\title{
Functional magnetic resonance imaging correlates of memory encoding in relation to achieving remission in first-episode schizophrenia ${ }^{\dagger}$
}

Michael Bodnar, Amelie M. Achim, Ashok K. Malla, Ridha Joober, Audrey Benoit and Martin Lepage

\section{Background}

Previous studies in schizophrenia have shown a strong relationship between memory deficits and a poor clinical outcome. However, no previous study has identified the functional neural correlates of memory encoding in relation to remission.

\section{Aims}

To determine whether functional magnetic resonance imaging (fMRI) activation patterns differed between individuals that later achieved remission $v$. those who did not.

\section{Method}

Forty-two participants with first-episode schizophrenia were divided into two groups after 1 year of treatment as per the 2005 remission in schizophrenia consensus definition. We then examined fMRI activation using three contrasts (associative $v$. item-oriented strategy, semantically unrelated $v$. related image pairs, and successful $v$. unsuccessful memory encoding) among 15 participants who had achieved remission (remitted group), 27 who had not (non-remitted group) and 31 healthy controls (control group).

\section{Results}

Participants in the non-remitted group displayed a positive activation in the posterior cingulate compared with those in the remitted group when encoding related images; no significant differences between the two groups were identified for the other contrasts. From the behavioural data, compared with the remitted and control groups, the non-remitted group demonstrated an inability to encode related images and displayed worse recognition memory overall.

\section{Conclusions}

This is the first study to identify differential neural activation between individuals with first-episode schizophrenia that later achieved remission $v$. those who did not. The behavioural and functional results together add to the growing evidence relating a poor clinical outcome in schizophrenia to memory-related deficits.

\section{Declaration of interest}

M.L. reports having received honorariums for educational events from Janssen-Ortho and Lilly. A.K.M. reports having received financial assistance/compensation for research and educational activities from Pfizer, Janssen-Ortho, AstraZeneca and Bristol-Myers Squibb. R.J. reports having received consultancy honorarium from Pfizer and Janssen-Ortho.
Memory encoding has been implicated as the key failure point in schizophrenia, ${ }^{1}$ with differential patterns of functional magnetic resonance imaging (fMRI) activation identified in the prefrontal cortex and medial temporal lobe in addition to the posterior cingulate and other midline areas. ${ }^{2,3}$ Moreover, the memory deficits observed in schizophrenia have been consistently related to a poorer functional ${ }^{4}$ and clinical ${ }^{5-8}$ outcome. Yet, no previous study has explicitly examined the functional relationship between memory encoding and outcome. A better understanding of this relationship could help improve our understanding of the pathophysiology of schizophrenia ${ }^{9,10}$ and aid in the development of newer, target-specific treatments. . $^{8,9,11,12}$

Using fMRI, we explored for activation differences among individuals with remitted and non-remitted first-episode schizophrenia (the remitted and non-remitted groups respectively) and healthy controls (the control group); remission was defined following the proposed 2005 consensus criteria. ${ }^{13}$ The fMRI paradigm used is sensitive to detecting activity in the prefrontal cortex, medial temporal lobe and midline posterior regions using three different contrasts: (a) associative $v$. itemoriented encoding strategy, (b) encoding semantically unrelated $v$. related image pairs, and (c) successful $v$. unsuccessful memory encoding. ${ }^{14,15}$ Previous work from our laboratory identified verbal

†See editorial, pp. 270-272, this issue. memory deficits ${ }^{5,6}$ and reduced grey matter in the medial temporal lobe bilaterally (parahippocampal gyrus $^{5}$ and hippocampus tail ${ }^{16}$ ) as markers of a poor clinical outcome. Based on these results, together with our previous fMRI result that identified reduced medial temporal lobe activation in participants with a first-episode of psychosis using the semantic relatedness contrast, ${ }^{15}$ we hypothesised that the non-remitted group, compared with the remitted and control groups, would show a selective deactivation in the medial temporal lobe for this particular contrast. Furthermore, regarding the fMRI behavioural data, we hypothesised that the non-remitted group would display a poorer recognition memory compared with both the remitted and the control groups.

\section{Method}

\section{Participants and treatment protocol}

All the participants with schizophrenia were treated at the Douglas Mental Health University Institute in Montreal, Canada, at the Prevention and Early Intervention Program for Psychoses (PEPP) - a specialised service providing treatment to individuals aged 14-30 years from a local catchment area with either affective or non-affective psychosis. Individuals with an IQ higher than 70 who had not taken antipsychotic medication for more than 1 month were consecutively admitted as in- or out-patients. See 
Malla et $a l^{17}$ (or visit www.douglas.qc.ca/pages/view?section _id=165) for more details.

For the neuroimaging study, only individuals aged 18-30 years with no previous history of neurological disease or head trauma causing loss of consciousness were eligible. In all, 45 individuals with first-episode schizophrenia (treated January 2004 to December 2008) had usable fMRI data. Three individuals were subsequently removed because of: excessive movement during scanning $(n=1)$ and failure to follow encoding instructions ( $n=2$; fMRI behavioural results below chance level). The remaining 42 participants were separated into two groups, the 15 who achieved remission $(35.7 \%)$ and the 27 who did not. Remission was defined as mild (3) or less on eight key symptoms of the Positive and Negative Syndrome Scale ${ }^{18}$ (PANSS; delusions, conceptual disorganisation, hallucinatory behaviour, blunted affect, social withdrawal, lack of spontaneity and flow of conversation, mannerisms and posturing, and unusual thought content) maintained for 6 consecutive months (in our case, from month 6 to 12 after admission). ${ }^{13}$

Diagnoses included schizophrenia (remitted group $n=12$, non-remitted group $n=21$ ), schizoaffective disorder (remitted group $n=2$, non-remitted group $n=6$ ) and schizophreniform disorder (remitted group $n=1$ ) according to the Structured Clinical Interview for DSM-IV ${ }^{19}$ confirmed between two senior research psychiatrists (A.K.M. and R.J.). Thirty-one healthy controls were recruited through advertisements in local newspapers and were included only if they had no current or previous history of (a) any Axis I disorders, (b) any neurological diseases, (c) head trauma causing loss of consciousness, and (d) a first-degree family member with schizophrenia or related schizophrenia-spectrum psychosis. See the online supplement for a description of the sociodemographic and clinical data collected.

After a comprehensive description of the study, written informed consent was obtained from all participants. Research protocols were approved by the McGill University Faculty of Medicine review board.

\section{fMRI task}

Stimuli consisted of pairs of clipart images (representing common objects, food or animals) arranged side by side on a screen presented through a projector and mirror system. Each trial consisted of an encoding cue presented for $3 \mathrm{~s}$, then a pair of images for $3 \mathrm{~s}$, followed by a fixation cross lasting 1 to $4 \mathrm{~s}$ (times randomly mixed between trials). The cues oriented the participant to perform one of two different encoding tasks: half promoted an associative strategy (compare images and select the one that was bigger in real-life size) and half promoted a deep item-oriented strategy (examine images and answer if at least one image represented a living entity); the encoding strategy contrast. Each of the two fMRI encoding sessions comprised of 56 different pairs of images: 28 semantically related (e.g. band-aid and medical kit) and 28 semantically unrelated (e.g. t-shirt and lizard); the semantic relatedness contrast. Semantic relatedness (unrelated and related) and encoding strategy (associative and item-oriented) were mixed randomly. See online Fig. DS1 for a pictorial representation of the task.

Following encoding, and while still in the scanner, recognition memory was assessed in two sessions by presenting all of the previously encoded pairs (intact and not rearranged) along with an equal number of distracter, never-seen-before pairs (half semantically related and half unrelated), for a total of 112 pairs per session. Participants were asked to respond to each pair as either old or new. The answers to the old pairs were used to distinguish successful encoding (old pairs recognised as such) from unsuccessful encoding (old pairs recognised as new); the subsequent memory effect contrast. Participants were told prior to encoding that recognition memory would be subsequently tested. Full details are published elsewhere. ${ }^{14,15}$

\section{fMRI data acquisition, processing and statistical analyses}

Scanning was carried out at the Montreal Neurological Institute on a 1.5T Siemens whole-body MRI system. Functional $T_{2}{ }^{*}$-weighted echo-planar images were acquired with blood oxygenation level-dependent contrast (repetition time $(\mathrm{TR})=$ $2130 \mathrm{~ms}$, echo time $(\mathrm{TE})=50 \mathrm{~ms}$, flip angle $=90^{\circ}$ ) covering the entire brain (25 interleaved slices parallel to the anteriorposterior commissural plane; voxel size $4 \times 4 \times 5 \mathrm{~mm}$ ); 214 volumes were acquired in each session. See online supplement for information about the participants' medication at the time of the scan.

Functional data were analysed using SPM2 on a Windowsbased workstation. For preprocessing, images were time corrected to account for differences in sampling times for different slices, motion corrected, spatially normalised (final voxel size $2 \times 2 \times 2 \mathrm{~mm}$ ) and smoothed with an $8 \mathrm{~mm}$ full-width at half maximum (FWHM) Gaussian kernel. Low-frequency temporal drifts were removed by applying a high-pass filter. The movement correction logs (obtained from the realignment procedure) were examined to ensure none of the participants had movements greater than $5 \mathrm{~mm}$ or $5^{\circ}$; one participant in the non-remitted group met that threshold and was removed. Data were analysed by the general linear model, in which individual events were modelled by a canonical haemodynamic response function.

Two design matrices were created for each participant: one for the encoding strategy and semantic relatedness contrasts and one for the subsequent memory effect contrast. The design matrices were modelled using correct and incorrect trials from both encoding sessions (see below for exceptions) differing in event types modelled. For the first matrix, four event types were modelled: (a) associative encoding of related pairs, (b) associative encoding of unrelated pairs, (c) item-oriented encoding of related pairs, and (d) item-oriented encoding of unrelated pairs; the six movement parameters were included as covariates. Only one of the two encoding sessions was included for two individuals (one each in the remitted and non-remitted groups) because only one session was completed; one participant in the non-remitted group was removed because of technical problems in acquiring responses. This amounted to 26 individuals in the non-remitted group, 15 in the remitted group and 31 in the control group.

For the second matrix, two event types were modelled: (a) successful encoding and (b) unsuccessful encoding; the six movement parameters were included as covariates. Only one of the two encoding sessions was included for one person in the remitted group because only one session was completed; two participants (one each in the remitted and non-remitted groups) were removed as the recognition task was not completed. This amounted to 26 individuals in the non-remitted group, 14 in the remitted group and 31 in the control group.

In all, three fMRI contrasts - encoding strategy (associative > item-oriented), semantic relatedness (unrelated $>$ related), and subsequent memory effect (successful > unsuccessful) - were compared between (a) remitted and non-remitted groups, (b) non-remitted and control groups, and (c) remitted and control groups. To correct for multiple comparisons, we applied a cluster extent threshold determined by Monte Carlo simulation using software written by Slotnick ${ }^{20,21}$ employing the following parameters: matrix $64 \times 64$, slices 25 , original voxel dimension $4 \times 4 \times 5$, resampled voxel resolution $2 \times 2 \times 2$, smoothing $8 \mathrm{~mm}$, 
$P$-corrected $=0.01, P$-voxel $=0.001$. After 10000 iterations, an extent threshold of 100 contiguous voxels was determined; this procedure prevented a false-positive rate above $1 \%$ due to multiple testing. Cluster-based thresholding is an alternative to the voxelbased correction and is often more sensitive to activation when one can reasonably expect multiple contiguous activated voxels. ${ }^{22,23}$ For significant clusters, the parameter estimate of each voxel was extracted using EasyROI, a SPM2 utility created by Pernet (www.sbirc.ed.ac.uk/cyril/cp_download.html), and averaged to provide an overall measure of activation for that cluster. Peak voxel coordinates were converted from Montreal Neurological Institute $(\mathrm{MNI})$ to Talairach using a non-liner transformation (http://imaging.mrc-cbu.cam.ac.uk/imaging/MniTalairach), with structures identified using Talairach Client (www.talairach.org/ client.html).

For the behavioural data, performance means and response times (encoding and recognition separately) were explored using a MANOVA, with group membership (non-remitted, remitted, control) as the between-group factor and encoding strategy (associative, item-oriented) and semantic relatedness (unrelated pairs, related pairs) as the within-group factors. Encoding performance was calculated as the percentage of correct answers to the encoding questions. One individual in the healthy control group performed at chance level for item-oriented encoding and was excluded. During debriefing, this participant reported giving a positive response only when both items were alive (only one needed to be alive). Since the distinction between associative and deep item-oriented encoding was not compromised by this response pattern, this participant was included in the fMRI analyses.

Recognition performance was calculated as: hit rate $(\mathrm{HR})=($ hits +0.5$) /($ number of items +1$)$ to evaluate the effect of encoding strategy on subsequent retrieval. Furthermore, to account for false alarms (FA), a discrimination index $(\mathrm{Pr}=\mathrm{HR}-$ $\mathrm{FA})$ and response bias $(\mathrm{Br}=(\mathrm{FA} /(1-\mathrm{Pr}))-0.5)$ (subtraction of 0.5 allowed an absence of bias to correspond to a value of 0 ) were calculated according to the two-high threshold model. ${ }^{24}$ The discrimination index provides an unbiased estimate of memory accuracy; $\mathrm{Br}$ is an index of the overall tendency to respond 'old' or 'new' regardless of accuracy - positive values indicate a familiarity bias (i.e. a tendency to say old) and negative values indicate a novelty bias (i.e. a propensity to say new). For discrimination index and response bias, only semantic relatedness and group membership were included in the analyses, as encoding strategy (associative $v$. item) was only part of the encoding phase. All performance data were $\log$ transformed to achieve normality; response time data were normally distributed. All analyses were two-tailed with a critical $P$-value of 0.05 and performed using SPSS version 12 on a Windows-based workstation.

\section{Results}

\section{Sociodemographic and clinical}

The three groups did not significantly differ in age, parental socioeconomic status, gender or handedness. The remitted and non-remitted groups had significantly fewer years of education and a lower full-scale IQ compared with the control group; outcome groups did not differ (Table 1). Outcome groups did not differ in positive or negative symptoms at first assessment but the remitted group showed significantly lower totals at month 6 and 12, as expected. Total antipsychotic dosage (in chlorpromazine equivalents) was similar at first assessment but was higher (non-significant) for the non-remitted group at months 6 and 12. At the time of the scan, the non-remitted group had significantly higher positive and negative symptom totals and were receiving a higher (non-significant) dosage of antipsychotic medication. Medication adherence did not differ at any time point (online Table DS1).

\section{Behavioural fMRI results}

There were significant (group semantic relatedness) interactions for encoding accuracy and for recognition hit-rate and response times; there were no other significant group-related interactions. Of note, for encoding accuracy, the remitted and control groups responded more accurately to related pairs than to unrelated pairs, whereas the non-remitted group displayed no such difference. Other analyses revealed that the remitted and non-remitted groups did not significantly differ in any instance but various between-group differences with the control group were present (Table 2). For discrimination index, the non-remitted group displayed poorer overall recognition memory compared with both the remitted and control groups. For response bias, it was revealed that all participants showed a stronger novelty bias (propensity to say 'new') when recognising related pairs of images over unrelated pairs of images (Table 3). See online supplement for a complete description of results.

\section{fMRI results}

For the semantic relatedness contrast (unrelated $>$ related) (Table 4 and Fig. 1), the 'remitted > non-remitted' comparison identified reduced activation in the left posterior cingulate in the non-remitted group. However, the parameter estimates from this cluster revealed that the non-remitted group actually had positive activation when encoding related pairs over unrelated pairs $\left(t_{25}=3.33, P=0.003\right)$; in contrast, the remitted group had negative activation when encoding related pairs over unrelated pairs $\left(t_{14}=-3.32, P=0.005\right)$.

Table 1 Sociodemographic characteristics for the non-remitted and remitted first-episode schizophrenia groups and the control group

\begin{tabular}{|c|c|c|c|c|c|c|c|}
\hline & \multirow{2}{*}{$\begin{array}{l}\text { Non-remitted group } \\
\qquad(n=27)\end{array}$} & \multirow{2}{*}{$\begin{array}{l}\text { Remitted group } \\
\qquad(n=15)\end{array}$} & \multirow{2}{*}{$\begin{array}{l}\text { Control group } \\
\qquad(n=31)\end{array}$} & \multicolumn{4}{|c|}{ Analysis } \\
\hline & & & & $F$ & $\chi^{2}$ & d.f. & $P$ \\
\hline Age at scan, years: mean (s.d.) & $23.7(4.2)$ & $24.2(3.0)$ & $24.7(3.3)$ & 0.59 & & 2,70 & 0.558 \\
\hline Parental socioeconomic status, ${ }^{a}$ mean (s.d.) & $3.4(1.2)$ & $3.3(1.3)$ & $3.1(1.0)$ & & 1.51 & 2 & 0.470 \\
\hline Education level, ${ }^{\mathrm{b}}$ mean (s.d.) & $11.4(2.2)$ & $12.6(3.1)$ & $14.8(2.1)$ & 14.94 & & 2,70 & $<0.001$ \\
\hline Full-scale IQ, ${ }^{\mathrm{C}}$ mean (s.d.) & 96.9 (14.3) & $98.7(15.7)$ & $109.0(12.6)$ & 6.67 & & 2,68 & 0.005 \\
\hline Male, $n(\%)$ & $21(77.8)$ & $10(66.7)$ & $17(54.8)$ & & 3.38 & 2 & 0.185 \\
\hline Right handed, $n(\%)$ & $20(74.1)$ & $12(80.0)$ & $29(93.5)$ & & 4.16 & 2 & 0.125 \\
\hline \multicolumn{8}{|c|}{$\begin{array}{l}\text { a. Hollingshead socioeconomic status, in which one is highest and five is lowest. } \\
\text { b. Number of years completed. Tukey's honestly significant difference tests revealed: non-remitted group }=\text { remitted group }(P=0.284) \text {; non-remitted group }<\text { control group }(P<0.001) \\
\text { remitted group }<\text { control group }(P=0.012) \text {. } \\
\text { c. Measured with the Wechsler Adult Intelligence Scale (WAIS-III) }{ }^{25} \text { data were available for only } 28 \text { individuals in the control group. Tukey's honestly significant difference tests } \\
\text { revealed: non-remitted group = remitted }(P=0.917) \text {; non-remitted group }<\text { control group }(P=0.006) \text {; remitted group }=\text { control group }(P=0.060) \text {. }\end{array}$} \\
\hline
\end{tabular}




\begin{tabular}{|c|c|c|c|c|}
\hline & \multicolumn{2}{|c|}{ Associative strategy } & \multicolumn{2}{|c|}{ Item-oriented strategy } \\
\hline & Unrelated, mean (s.d.) & Related, mean (s.d.) & Unrelated, mean (s.d.) & Related, mean (s.d.) \\
\hline \multicolumn{5}{|l|}{ Encoding } \\
\hline \multicolumn{5}{|l|}{ Accuracy $^{a}$} \\
\hline Non-remitted group ${ }^{b}$ & $0.91(0.07)$ & $0.91(0.09)$ & $0.84(0.12)$ & $0.88(0.11)$ \\
\hline Remitted group ${ }^{b}$ & $0.86(0.09)$ & $0.94(0.07)$ & $0.79(0.22)$ & $0.84(0.22)$ \\
\hline Control group ${ }^{b}$ & $0.93(0.07)$ & $0.96(0.05)$ & $0.91(0.08)$ & $0.96(0.08)$ \\
\hline \multicolumn{5}{|l|}{ Response time, ms } \\
\hline Non-remitted group & 1508 (190) & $1510(166)$ & $1504(252)$ & 1387 (177) \\
\hline Remitted group & $1545(182)$ & 1599 (160) & 1485 (220) & 1435 (243) \\
\hline Control group & $1423(205)$ & $1436(193)$ & $1351(207)$ & 1248 (199) \\
\hline \multicolumn{5}{|l|}{ Recognition } \\
\hline \multicolumn{5}{|l|}{ Hit rate ${ }^{a}$} \\
\hline Non-remitted group ${ }^{b}$ & $0.73(0.15)$ & $0.78(0.15)$ & $0.65(0.18)$ & $0.70(0.19)$ \\
\hline Remitted group ${ }^{b}$ & $0.80(0.11)$ & $0.85(0.09)$ & $0.69(0.15)$ & $0.74(0.15)$ \\
\hline Control group ${ }^{b}$ & $0.85(0.07)$ & $0.87(0.06)$ & $0.79(0.10)$ & $0.78(0.09)$ \\
\hline \multicolumn{5}{|l|}{ Response time, ms } \\
\hline Non-remitted group & $1395(267)$ & 1267 (271) & $1360(265)$ & $1313(286)$ \\
\hline Remitted group & 1359 (318) & 1198 (237) & $1448(255)$ & $1223(301)$ \\
\hline Control group & 1158 (191) & $1071(157)$ & 1209 (194) & 1102 (184) \\
\hline
\end{tabular}

\begin{tabular}{|c|c|c|c|c|}
\hline \multirow[b]{2}{*}{ Performance $^{a}$} & \multicolumn{2}{|c|}{ Discrimination index } & \multicolumn{2}{|c|}{ Response bias } \\
\hline & Unrelated, mean (s.d.) & Related, mean (s.d.) & Unrelated, mean (s.d.) & Related, mean (s.d.) \\
\hline Non-remitted group ${ }^{\mathrm{b}}$ & $0.56(0.23)$ & $0.63(0.26)$ & $-0.23(0.18)$ & $-0.29(0.25)$ \\
\hline Remitted group $^{b}$ & $0.68(0.13)$ & $0.76(0.13)$ & $-0.28(0.20)$ & $-0.38(0.17)$ \\
\hline Control group ${ }^{\mathrm{b}}$ & $0.75(0.10)$ & $0.78(0.08)$ & $-0.21(0.18)$ & $-0.32(0.16)$ \\
\hline
\end{tabular}
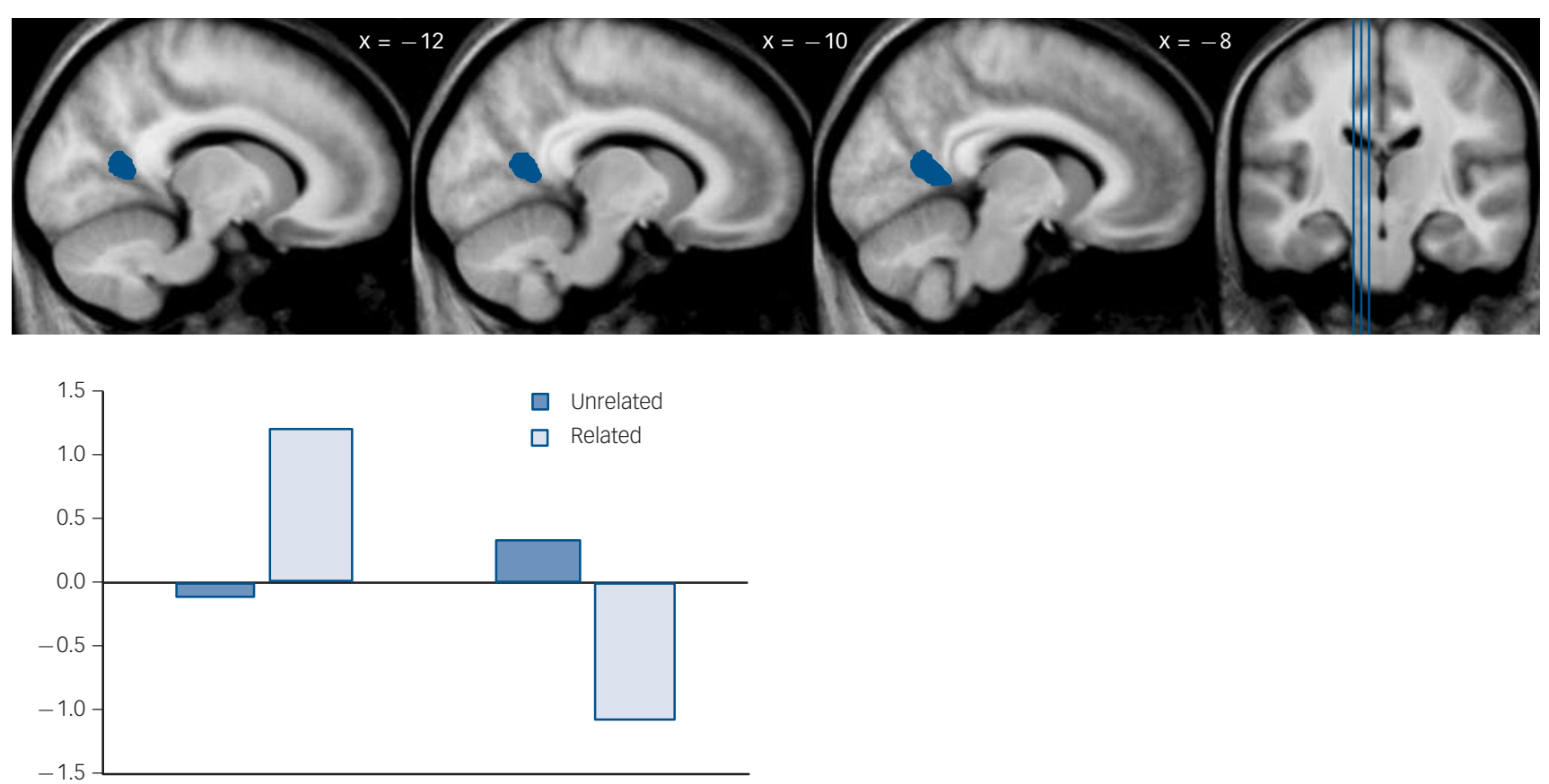

Non-remitted group

Remitted group

Fig. 1 Result of 'remitted > non-remitted' group comparison for semantic relatedness contrast ('unrelated pairs > related pairs').

Between-group analysis revealed that the left posterior cingulate was significantly more active in the non-remitted group relative to the remitted group when encoding semantically related images $\left(P<0.01\right.$, corrected). Result is superimposed on an average $T_{1}$ magnetic resonance template image. The location of the three sagittal sections is illustrated on the coronal plane, with $x$-coordinates presented in Talairach space. The bar graph indicates the parameter estimates during encoding in each outcome group. 


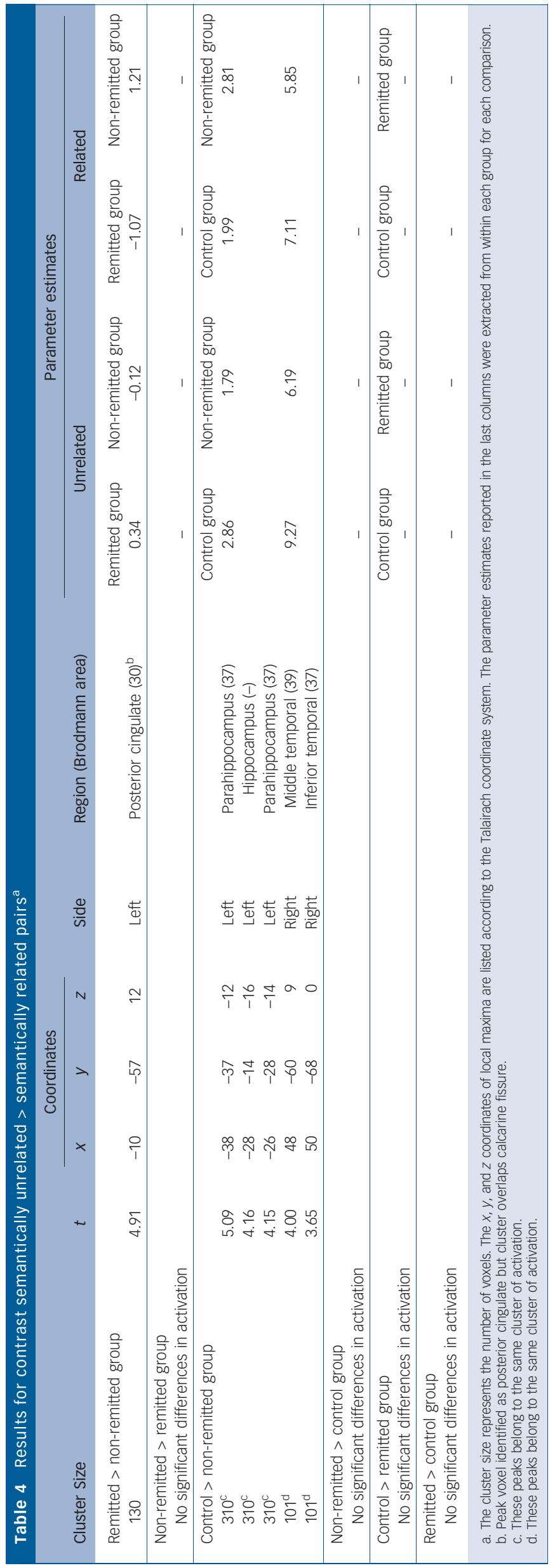

The 'non-remitted > remitted' comparison identified no significant differences. The 'control > non-remitted' comparison identified reduced activation in the non-remitted group in the left parahippocampus/hippocampus and right inferior/middle temporal gyrus. Parameter estimates from the parahippocampus/hippocampus cluster revealed that the non-remitted group had a larger positive activation when encoding related pairs over unrelated pairs in the parahippocampus $\left(t_{25}=4.24, P<0.001\right)$. In contrast, the control group had a larger positive activation when encoding unrelated pairs over related pairs $\left(t_{30}=-3.27\right.$, $P=0.003)$. For the inferior/middle temporal cluster, the control group had a larger positive activation when encoding unrelated pairs over related pairs $\left(t_{30}=-6.45, P<0.001\right)$, whereas the non-remitted group did not differ in the same pattern of activation $\left(t_{25}=-1.23, P=0.231\right)$. The 'non-remitted $>$ control' comparison as well as the 'control $>$ remitted' and 'remitted $>$ control' comparisons identified no significant differences.

For the encoding strategy contrast (associative > itemoriented) (Table DS2), significant differences were identified for only the 'control > remitted' comparison, which identified reduced activation in the right precuneus and bilateral middle cingulate in the remitted group. Parameter estimates from the precuneus revealed that the remitted group had positive activation for item-oriented encoding over associative encoding $\left(t_{14}=-6.90, P<0.001\right)$; the control group did not differ in larger positive activation for item-oriented over associative encoding $\left(t_{30}=-1.74, P=0.092\right)$. The remitted group had a larger negative activation for associative encoding over item-oriented encoding in the middle cingulate $\left(t_{14}=-4.49, P=0.001\right)$, whereas the control group did not significantly differ in negative activation for associative or item-oriented encoding $\left(t_{30}=0.15, P=0.884\right)$.

For the subsequent memory effect contrast (successful > unsuccessful) (online Table DS3), significant differences were observed for only the 'non-remitted > control' comparison, which identified reduced activation in the left superior temporal gyrus/ insula in the control group. Parameter estimates from this cluster revealed that the control group had a larger negative activation for successful encoding over unsuccessful encoding $\left(t_{30}=-5.12\right.$, $P<0.001$ ), whereas the non-remitted group did not differ in the larger negative activation for unsuccessful encoding over successful encoding $\left(t_{25}=1.64, P=0.114\right)$.

\section{Supplementary analyses}

The 'non-remitted > remitted' comparison for the semantic relatedness contrast was re-analysed including covariates with only minimal changes to the posterior cingulate result: handedness, smaller cluster size (122 voxels) and $t$-value (4.88); antipsychotic dosage at scan, smaller cluster size (124 voxels) but larger $t$-value (4.93); IQ, larger cluster size (137 voxels) and $t$-value (5.30); and the three covariates together, smaller cluster size (123 voxels) but larger $t$-value (5.07).

The parameter estimates (related and unrelated) from the left posterior cingulate were correlated with the eight symptoms from the remission definition using Spearman's correlations (critical $P$-value set at $0.006(0.05 / 8)$; Bonferroni corrected). There were no notable correlations with symptom ratings at time of scan. Activation values were also correlated with encoding accuracy (critical $P<0.025,(0.05 / 2)$ ) and discrimination index (critical $P<0.017,(0.05 / 3)$ ) using Pearson's correlation, but no significant relationships were found (online Table DS4).

\section{Discussion}

Using three fMRI contrasts: encoding strategy (associative $>$ itemoriented), semantic relatedness (unrelated $>$ related), and 
subsequent memory effect (successful $>$ unsuccessful), we explored for functional differences during memory encoding among participants with first-episode schizophrenia who later achieved remission $v$. those who did not and healthy controls.

Between outcome groups, the non-remitted group had a positive activation in the left posterior cingulate when encoding semantically related images over unrelated images, whereas those in the remitted group had a negative activation. No significant differences were identified using the encoding strategy or subsequent memory effect contrasts. For comparisons with the control group, for semantic relatedness, the non-remitted group had a larger positive activation when encoding related images over unrelated images in the left medial temporal lobe, whereas the control group had a larger positive activation when encoding unrelated images over related images. Both the non-remitted and control groups had larger positive activation for unrelated images over related images activation in the right temporal gyrus (non-significant in the non-remitted group). For encoding strategy, the remitted group had a larger negative activation in the middle cingulate bilaterally for associative encoding over item-oriented encoding, whereas the control group had no significant activation differences between encoding strategies. The remitted group also had a negative activation in the right precuneus for associative encoding over item-oriented encoding, whereas the control group had a positive activation. For the subsequent memory effect, controls had a larger negative activation in the left superior temporal gyrus for successful encoding over unsuccessful encoding, whereas the non-remitted group had a larger negative activation (non-significant) for unsuccessful encoding over successful encoding.

For the fMRI behavioural data, results were limited to semantic relatedness. During encoding, the remitted and control groups responded more accurately to related images over unrelated images, whereas the non-remitted group showed no difference suggesting an impaired use of semantic encoding. For recognition, the non-remitted group performed significantly worse in overall recognition memory when accounting for false alarms compared with both the remitted and the control groups. This supported the growing literature relating worse memory performance to a poor clinical outcome in schizophrenia. ${ }^{5-8}$

\section{Semantic processing, positive activity and clinical outcome}

There is little doubt semantic processing is impaired in schizophrenia, with impairments ranging from a lack of knowledge about people or objects to inabilities to form relationships between objects (or images). ${ }^{26-28}$ More specifically regarding the latter, a recent meta-analysis of studies involving event-related potentials found that people with schizophrenia are more impaired when processing related conditions (words or images) compared with unrelated conditions. ${ }^{26}$ This failure to process related images properly was supported by our results but was more particular to those participants who did not achieve remission. Behaviourally, the non-remitted group showed no advantage when encoding related images compared with the remitted and control groups.

Functionally, the non-remitted group had a positive activation in the left posterior cingulate cortex when encoding semantically related images over unrelated images; the remitted group had a negative activation. In addition, the non-remitted group had a larger positive activation in the left medial temporal lobe when processing related images over unrelated images, whereas the results were the opposite in the control group. Although a positive activation in the posterior cingulate cortex or medial temporal lobe seemed counterintuitive at first and was at variance with our initial hypothesis, several studies have associated positive (or increased) activity in the posterior cingulate cortex and medial temporal lobe with memory dysfunction in schizophrenia.

In schizophrenia, the posterior cingulate cortex has been shown to remain hyperactive when mentally engaged ${ }^{29-31}-$ the opposite of what is known to occur as per the default-mode network ${ }^{29,32}$ - with this hyperactivity associated with worse memory performance. ${ }^{29,30}$ With support from our main findings, we suggest that the posterior cingulate cortex remains hyperactive in the non-remitted group during an attention-oriented task (encoding related images) resulting in improper encoding and poorer memory. However, we found posterior cingulate cortex activity did not correlate with encoding accuracy or recognition memory (discrimination index). Nevertheless, the posterior cingulate cortex is believed to be crucial to memory encoding ${ }^{28,33}$ and semantic processing ${ }^{28}$ but how it may be functionally related to clinical outcome requires further exploration. Regarding the medial temporal lobe, a recent fMRI meta-analysis of memory function in schizophrenia showed increased activity in the left parahippocampal gyrus during encoding. ${ }^{2}$ The authors suggested that in schizophrenia, the increased activity during encoding may result from inefficient, compensatory brain activity until a suitable encoding strategy is reached. ${ }^{34}$ So, during encoding, it appears the non-remitted group may not be fully engaging the correct encoding mechanisms (left parahippocampal gyrus) when trying to encode related images. However, since parahippocampal activity only differed with the control group and not the remitted group, functional activity of this structure, as per memory encoding, may not be related to clinical outcome directly. More studies are required to explore this potential relationship.

Our results support that functional impairments exist in people with schizophrenia when processing related images but further demonstrate that these impairments may be specific to or driven by individuals with a poorer outcome. It would be interesting to explore if semantically related N400 differences ${ }^{26}$ exist between the remitted and non-remitted groups.

\section{Other functional differences with healthy controls}

Compared with the control group, the non-remitted group had increased activity in the left superior temporal gyrus/insula for the subsequent memory effect contrast but nothing identified for the encoding strategy contrast. In contrast, the remitted group showed decreased activity in the right precuneus and bilateral middle cingulate for the encoding strategy contrast but nothing identified for the subsequent memory effect contrast. As with our previous study, we found limited results for both of these particular contrasts. ${ }^{15}$ However, we did not use an associative memory recognition task (which typically requires the participant to distinguish between intact and rearranged pairs of items) and therefore could not examine associative $v$. item memory during retrieval. Perhaps a real associative $v$. item task would have shown more significant group differences both at the behavioural and neuronal levels for these two contrasts.

\section{Implications}

We identified increased activity in the posterior cingulate cortex in the non-remitted group compared with the remitted group specific to semantic processing during encoding. This finding suggested that the functional activity of semantic processing may be sensitive to detecting differences within a patient sample (i.e. good $v$. poor outcome). As a region of interest, the posterior cingulate cortex should be explored further both structurally and 
functionally in relation to psychopathology for a better understanding of any underlying relationships. It could also be targeted using pharmaco-fMRI techniques ${ }^{35,36}$ in relation to the numerous receptor abnormalities (glutamate, gammaaminobutyric acid and muscarnic) identified in schizophrenia ${ }^{37,38}$ to expand the research into developing newer, target-specific treatments for people with schizophrenia in the hope of achieving a better outcome.

\section{Limitations}

First, ethical approval allowed for a first-assessment scan and follow-up scans at 1 year and 2 years thereafter, with fMRI data collected only during the first-assessment scan. Thus, scanning could not take place when remission status was determined after 1 year of treatment. At the time of the fMRI scan, 1 of the 15 participants in the remitted group was in a non-remitted state, whereas 10 of the 27 participants in the non-remitted group were in a remitted state. Re-analysing the data with participants separated based on clinical data near the time of scan showed similar results. Of interest, the posterior cingulate cortex (cluster: 68 voxels, $t$-value $=4.27$ ) was still hyperactive in the non-remitted group when encoding related images. Since the majority of the participants had obtained their 1-year remission status by the time of the fMRI scan, we feel our results would be similar had scanning taken place at month 12 .

Second, not finding increased activity in the posterior cingulate cortex in the non-remitted $v$. control group or increased activity in the medial temporal lobe in the non-remitted $v$. remitted group may have limited the interpretation of our results. However, these activations were in fact present but were not detected due to our conservative threshold. Upon lowering the threshold $(P<0.005$, uncorrected), we found that the nonremitted group had positive activity in the left posterior cingulate cortex ( $x=-6, y=-48, z=6$, cluster: 222 voxels) compared with the control group and positive activity in the left parahippocampal gyrus ( $x=-28, y=-28, z=-19$, cluster: 51 voxels) compared with the remitted group when encoding related pairs over unrelated pairs. However, these results may be a false-positive because of the low threshold used. Nevertheless, the non-remitted group do show altered activity compared with the remitted and control groups in both the posterior cingulate cortex and medial temporal lobe, supporting our initial interpretations.

Third, although the inclusion of medicated and relatively stable participants could imply that medication may have affected the cognitive processes being studied and limited the generalisability of our findings to unmedicated individuals with first-episode schizophrenia, including people who are stable enough to comply with the imaging procedures is likely to produce more reliable results for fMRI studies of cognitive functions than by including unmedicated and symptomatically unstable individuals who are not able to follow the task instructions. Although antipsychotic medications have been shown to affect fMRI results, ${ }^{39,40}$ we demonstrated our main result was unaffected when including antipsychotic dosage as a covariate. Nevertheless, caution is warned when interpreting our results.

A final limitation was not incorporating the two-design matrices into one matrix to analyse the fMRI data for the subsequent memory effect. This would have been advantageous since we had an interaction between recognition memory and semantic relatedness for the behavioural data. However, we could not employ a single matrix including both 'successful $v$. unsuccessful memory encoding' and 'unrelated $v$. related image pairs' as there were not an equal number of events that could be modelled for all of the participants. Of those that could be modelled, our final sample size would have been reduced to 18 people in the control group, 10 in the remitted group and 18 in the non-remitted group. As such, we decided to explore only 'successful $v$. unsuccessful memory encoding' for the subsequent memory effect analysis.

\begin{abstract}
Michael Bodnar, PhD, Brain Imaging Group and Prevention and Early Intervention Program for Psychoses (PEPP - Montréal), Douglas Mental Health University Institute and Department of Neurology \& Neurosurgery, McGill University, Montréal; Amelie M. Achim, PhD, Brain Imaging Group, Douglas Mental Health University Institute, and Department of Neurology \& Neurosurgery, McGill University, Montréal; Ashok K. Malla, MD, Prevention and Early Intervention Program for Psychoses (PEPP - Montréal), Douglas Mental Health University Institute, and Department of Psychiatry, McGill University, Montréal; Ridha Joober, MD, PhD, Brain Imaging Group and Prevention and Early Intervention Program for Psychoses (PEPP - Montréal), Douglas Mental Health University Institute, and Department of Psychiatry, McGill University, Montréal; Audrey Benoit, BSC, Brain Imaging Group, Douglas Mental Health University Institute, Montréal; Martin Lepage, PhD, Brain Imaging Group and Prevention and Early Intervention Program for Psychoses (PEPP - Montréal), Douglas Mental Health University Institute, and Department of Neurology \& Neurosurgery and Department of Psychiatry, McGill University, Montréal, Canada
\end{abstract}

Correspondence: Martin Lepage, PhD, Douglas Mental Health University Institute, Frank B Common Pavilion, 6875 LaSalle Blvd, Verdun, Quebec H4H 1R3, Canada. Email: martin.lepage@mcgill.ca

First received 16 Jun 2011, final revision 28 Oct 2011, accepted 30 Nov 2011

\section{Funding}

This work was funded by operating grants from the Canadian Institutes of Health Research (CIHR; \#68961) and the Sackler Foundation to M.L. and A.K.M. M.L. is supported by a salary award from the Fonds de la recherche en santé du Québec (FRSQ). A.K.M. is supported by the Canada Research Chairs programme.

\section{Acknowledgements}

We thank the PEPP-Montreal research staff for their help with recruitment and clinical assessments of all participants involved.

\section{References}

1 Cirillo MA, Seidman L. Verbal declarative memory dysfunction in schizophrenia: from clinical assessment to genetics and brain mechanisms. Neuropsychol Rev 2003; 13: 43-77.

2 Ragland JD, Laird AR, Ranganath C, Blumenfeld RS, Gonzales SM, Glahn DC. Prefrontal activation deficits during episodic memory in schizophrenia. Am J Psychiatry 2009; 166: 863-74.

3 Achim AM, Lepage M. Episodic memory-related activation in schizophrenia: meta-analysis. Br J Psychiatry 2005; 187: 500-9.

4 Green MF. What are the functional consequences of neurocognitive deficits in schizophrenia? Am J Psychiatry 1996; 153: 321-30.

5 Bodnar M, Harvey P-O, Malla A, Joober R, Lepage M. The parahippocampal gyrus as a neural marker of early remission in first episode psychosis: a voxel-based morphometry study. Clin Schizophr Relat Psychoses 2011; 4 217-28.

6 Bodnar M, Malla A, Joober R, Lepage M. Cognitive markers of short-term clinical outcome in first-episode psychosis. Br J Psychiatry 2008; 193: 297-304.

7 Helldin L, Kane JM, Karilampi U, Norlander T, Archer T. Remission and cognitive ability in a cohort of patients with schizophrenia. J Psychiatr Res 2006; 40: 738-45.

8 Toulopoulou T, Murray RM. Verbal memory deficit in patients with schizophrenia: an important future target for treatment. Expert Rev Neurother 2004; 4: 43-52.

9 McGuire P, Howes OD, Stone J, Fusar-Poli P. Functional neuroimaging in schizophrenia: diagnosis and drug discovery. Trends Pharmacol Sci 2008; 29 91-8.

10 Saykin AJ, Gur RC, Gur RE, Mozley PD, Mozley LH, Resnick SM, et al. Neuropsychological function in schizophrenia. Selective impairment in memory and learning. Arch Gen Psychiatry 1991; 48: 618-24.

11 Hyman SE, Fenton WS. Medicine. What are the right targets for psychopharmacology? Science 2003; 299: 350-1. 
12 Ranganath C, Minzenberg MJ, Ragland JD. The cognitive neuroscience of memory function and dysfunction in schizophrenia. Biol Psychiatry 2008; 64 $18-25$.

13 Andreasen NC, Carpenter Jr WT, Kane JM, Lasser RA, Marder SR, Weinberger DR. Remission in schizophrenia: proposed criteria and rationale for consensus. Am J Psychiatry 2005; 162: 441-9.

14 Achim AM, Bertrand MC, Montoya A, Malla AK, Lepage M. Medial temporal lobe activations during associative memory encoding for arbitrary and semantically related object pairs. Brain Res 2007; 1161: 46-55.

15 Achim AM, Bertrand MC, Sutton H, Montoya A, Czechowska Y, Malla AK, et al. Selective abnormal modulation of hippocampal activity during memory formation in first-episode psychosis. Arch Gen Psychiatry 2007; 64: 999-1014.

16 Bodnar M, Malla AK, Czechowska $Y$, Benoit A, Fathalli F, Joober R, et al Neural markers of remission in first-episode schizophrenia: a volumetric neuroimaging study of the hippocampus and amygdala. Schizophr Res 2010; 122: $72-80$.

17 Malla A, Norman R, McLean T, Scholten D, Townsend L. A Canadian programme for early intervention in non-affective psychotic disorders. Aust N Z J Psychiatry 2003; 37: 407-13.

18 Kay S, Fiszbein A, Opler L. The positive and negative syndrome scale (PANSS) for schizophrenia. Schizophr Bull 1987; 13: 261-76.

19 First MB, Spitzer RL, Gibbon M, Williams JBW. Structured Clinical Interview for DSM-IV Axis I Disorders, Patient Edition (SCID-I/P \& SCID-I/NP), Version 2. New York Psychiatric Institute, Biometrics Research, 1998.

20 Slotnick SD, Moo LR, Segal JB, Hart Jr J. Distinct prefrontal cortex activity associated with item memory and source memory for visual shapes. Brain Res Cogn Brain Res 2003; 17: 75-82.

21 Slotnick SD, Schacter DL. A sensory signature that distinguishes true from false memories. Nat Neurosci 2004; 7: 664-72.

22 Forman SD, Cohen JD, Fitzgerald M, Eddy WF, Mintun MA, Noll DC. Improved assessment of significant activation in functional magnetic resonance imaging (fMRI): use of a cluster-size threshold. Magn Reson Med 1995; 33: 636-47.

23 Petersson KM, Nichols TE, Poline JB, Holmes AP. Statistical limitations in functional neuroimaging. II. Signal detection and statistical inference. Philos Trans R Soc Lond B Biol Sci 1999; 354: 1261-81.

24 Snodgrass JG, Corwin J. Pragmatics of measuring recognition memory: applications to dementia and amnesia. J Exp Psychol Gen 1988; 117 34-50.

25 Wechsler D. Wechsler Adult Intelligence Scale (3rd edn). The Psychological Corporation, 1997.

26 Wang K, Cheung EF, Gong QY, Chan RC. Semantic processing disturbance in patients with schizophrenia: a meta-analysis of the n400 component. PLOS One 2011; 6: e25435.
27 Doughty OJ, Done DJ. Is semantic memory impaired in schizophrenia? A systematic review and meta-analysis of 91 studies. Cogn Neuropsychiatry 2009; 14: 473-509.

28 Binder JR, Desai RH, Graves WW, Conant LL. Where is the semantic system? A critical review and meta-analysis of 120 functional neuroimaging studies. Cereb Cortex 2009; 19: 2767-96.

29 Whitfield-Gabrieli S, Thermenos HW, Milanovic S, Tsuang MT, Faraone SV, McCarley RW, et al. Hyperactivity and hyperconnectivity of the default network in schizophrenia and in first-degree relatives of persons with schizophrenia. Proc Natl Acad Sci USA 2009; 106: 1279-84.

30 Meda SA, Stevens MC, Folley BS, Calhoun VD, Pearlson GD. Evidence for anomalous network connectivity during working memory encoding in schizophrenia: an ICA based analysis. PLoS One 2009; 4: e7911.

31 Garrity AG, Pearlson GD, McKiernan K, Lloyd D, Kiehl KA, Calhoun VD Aberrant "default mode" functional connectivity in schizophrenia. Am J Psychiatry 2007; 164: 450-7.

32 Broyd SJ, Demanuele C, Debener S, Helps SK, James CJ, Sonuga-Barke EJ. Default-mode brain dysfunction in mental disorders: a systematic review. Neurosci Biobehav Rev 2009; 33: 279-96.

33 Wang L, Laviolette P, O'Keefe K, Putcha D, Bakkour A, Van Dijk KR, et al. Intrinsic connectivity between the hippocampus and posteromedial cortex predicts memory performance in cognitively intact older individuals. Neuroimage 2010; 51: 910-7.

34 Ramsey NF, Jansma JM, Jager G, Van Raalten T, Kahn RS. Neurophysiological factors in human information processing capacity. Brain 2004; 127: 517-25.

35 Honey G, Bullmore E. Human pharmacological MRI. Trends Pharmacol Sci 2004; 25: 366-74.

36 Stein EA. fMRI: a new tool for the in vivo localization of drug actions in the brain. J Anal Toxicol 2001; 25: 419-24.

37 Newell KA, Zavitsanou K, Huang XF. Ionotropic glutamate receptor binding in the posterior cingulate cortex in schizophrenia patients. Neuroreport 2005; 16: 1363-7.

38 Newell KA, Zavitsanou K, Jew SK, Huang XF. Alterations of muscarinic and GABA receptor binding in the posterior cingulate cortex in schizophrenia. Prog Neuropsychopharmacol Biol Psychiatry 2007; 31: 225-33.

39 Abbott C, Juarez M, white T, Gollub RL, Pearlson GD, Bustillo J, et al. Antipsychotic dose and diminished neural modulation: a multi-site fMRI study. Prog Neuropsychopharmacol Biol Psychiatry 2011; 35: 473-82.

40 Ettinger U, Williams SC, Fannon D, Premkumar P, Kuipers E, Moller HJ, et al. Functional magnetic resonance imaging of a parametric working memory task in schizophrenia: relationship with performance and effects of antipsychotic treatment. Psychopharmacology (Berl) 2011; 216: 17-27. 